\title{
Robust control in the quantum domain
}

\author{
Andrew Doherty ${ }^{1}$, John Doyle ${ }^{1}$, Hideo Mabuchi ${ }^{1}$, \\ Kurt Jacobs ${ }^{2}$, Salman Habib ${ }^{2}$ \\ ${ }^{1}$ California Institute of Technology, ${ }^{2}$ Los Alamos National Laboratory
}

\begin{abstract}
Recent progress in quantum physics has made it possible to perform experiments in which individual quantum systems are monitored and manipulated in real time. The advent of such new technical capabilities provides strong motivation for the development of theoretical and experimental methodologies for quantum feedback control. The availability of such methods would enable radically new approaches to experimental physics in the quantum realm. Likewise, the investigation of quantum feedback control will introduce crucial new considerations to control theory, such as the uniquely quantum phenomena of entanglement and measurement back-action. The extension of established analysis techniques from control theory into the quantum domain may also provide new insight into the dynamics of complex quantum systems. We anticipate that the successful formulation of an input-output approach to the analysis and reduction of large quantum systems could have very general applications in nonequilibrium quantum statistical mechanics and in the nascent field of quantum information theory.
\end{abstract}

\section{Introduction}

It would be of great interest in quantum physics to develop controlled and systematic methods for deriving approximate descriptions of complex dynamical systems. A range of powerful techniques that may be applicable to quantum scenarios have been previously investigated in the context of robust control theory; here we are interested in model reduction via balanced truncation in the Hankel norm [1]. Balanced truncation and related methods (e.g. based on the gap metric) are particularly attractive due to the availability of tight error bounds and for giving considerable insight into the interconnection of dynamical systems. Tractable schemes for quantum model reduction would have important applications in non-equilibrium statistical mechanics and in the development of quantum information technology - one of our main long-term goals will be to use model reduction to facilitate numerical simulation of fault-tolerant architectures for quantum computers [2]. This conference paper describes our first results on balanced truncation of coupled quantum systems and the formulation of the input-output descriptions of the dynamics of quantum error-correcting codes.

We anticipate that a general program of extending modern control-theoretic methods to quantum scenarios would have tremendous impact on experimental research in quantum physics as well [3]. In this context we are particularly interested in robust controller synthesis and controller reduction methodologies for feedback control of open quantum systems via real-time processing of measured output signals. New experimental techniques in the manipulation of quantum systems show the potential for genuinely quantum technologies such as quantum computers, however active feedback and control will be crucial to their functioning and motivate the adoption of control theory concepts and methods in quantum physics generally.

\section{Quantum Dynamics and Linear Systems Theory}

The first step in applying control-theoretic approaches to the approximation of quantum dynamics is to write the Schrödinger equation as a system of linear ordinary differential equations for an appropriate set of dynamical variables that characterize the quantum state. This set of variables will depend on the physical system, and more specifically on the parameters that are of most interest in a particular problem. One general approach in the spirit of the Shrödinger picture of quantum dynamics is to write the first order linear differential equations for the matrix elements of the quantum density operator and to separate those out into quantities of interest and quantities to be reduced. An approach more natural from the Heisenberg picture is to write the dynamics in terms of linear ordinary differential equations for operator expectation values - as long as a quorum of system observables is chosen this is precisely equivalent. A common example of such a quorum is the elements of the Bloch vector $(\langle X\rangle,\langle Y\rangle,\langle Z\rangle)$, that completely define the quantum state of a two-state system. The operators $X, Y, Z$ are the familiar Pauli operators.

It is often the case that only the reduced state of some subsystem is of interest. An important question is to what extent a simple description of the dynamics ('subdynamics') of this reduced system may be found. Here 
we consider the specific example of two coupled twostate systems ('spins'), where only the state of the first spin is of direct interest, and where the second spin is phase damped by coupling to an infinite reservoir. Although this is an almost trivial example and full numerical integration of the dynamics would pose no computational difficulty for such a low-dimensional problem, the procedures we follow are simple and systematic and may thus be applied to a wide range of more complex quantum systems of significant physical interest.

In our example, the state of the full system is described by the Bloch vectors of the two spins $\left(\left\langle I_{1}\right\rangle,\left\langle J_{2}\right\rangle, I, J=\right.$ $X, Y, Z)$ along with nine other quantities of the form $\left\langle I_{1} J_{2}\right\rangle$. The necessity of having so many 'interconnection variables' in this problem. immediately exemplifies one of the fundamental differences found in interconnecting quantum systems - the state space associated with the joint system is very much larger than would be the case for classical dynamics, as a result of the tensor product structure of the Hilbert space. Rather than having $3 N$ degrees of freedom (where 3 is the number of variables describing an isolated single spin system), a system made up of $N$ interacting spins will have $2^{N}-1$ degrees of freedom. The multitude of extra parameters characterizes the entanglement (quantum correlation) of the individual systems. (We recognize that the same dimensional arithmetic holds for the dynamics of classical probability distributions, but the quantum feature here is that no underlying 'trajectory' picture exists in which the state spaces may be combined by direct sum.) Hence, we expect model reduction techniques to be of great utility in the general study of interconnected quantum systems.

The quantities of interest in our example are a quorum of expectation values for a physical subsystem, namely the first spin. This partitioning of the variables according to physical subsystems is not the only type of problem that may be considered; it may be the case for example that the full system is simply one highdimensional system for which only a few expectation values are of interest, with other moments being important only as a means of calculating these. In any such scenario it is possible to proceed exactly as we do here. Another important consideration is that even for a system made up of many coupled subsystems it may not be the reduced density matrix for any given subsystem that is of primary interest. In quantum error-correcting codes, for example, it is the Bloch vector corresponding to the state of the logical (encoded) spin that is of interest, which is embedded within a highly entangled subspace of a string of real (physical) spins. Approximate simulations of realistic (imperfect) error correction protocols may well be facilitated by exactly the means described here, with an appropriate parametrization of the joint system state as is discussed below. In general, the selection of a particular set of parameters with which to describe the dynamics of the overall system must be based not only on knowledge of the dynamics and the Hilbert space structure of the system but also by what features of these dynamics are relevant to the question at hand.

Getting back to our example, we imagine that the two systems have a simple coupling and as we mentioned above the second spin suffers phase damping. If we did not introduce any dissipation the eigenvalues associated with our system of linear differential equations would all have real parts equal to zero (unitary dynamics). By adding the phase damping we guarantee that both the overall system and the subsystem on which the model reduction is performed are Hurwitz. Physically we are in any case most interested in applications to open quantum systems where there will almost always be some dissipative dynamics which usually ensures the stability of the equations of motion. Thus we consider the following master equation for the overall system,

$$
\begin{aligned}
\dot{\rho} & =-i[H, \rho]+\gamma \mathcal{D}\left[Z_{2}\right] \rho, \\
H & =\frac{1}{2} \hbar \omega_{1} Z_{1}+\frac{1}{2} \hbar \omega_{2} Z_{2}+\hbar g X_{1} X_{2},
\end{aligned}
$$

where for an arbitrary operator $c, \mathcal{D}[c] \rho=\bar{c} \rho c^{\dagger}-$ $\frac{1}{2} c^{\dagger} c \rho-\frac{1}{2} \rho c^{\dagger} c$. This operator differential equation can be converted to an equivalent set of ordinary differential equations for a set of operator expectation values by standard techniques [4]. Here we write only those equations that couple to the Bloch vector of the first spin:

$$
\begin{aligned}
\left\langle\dot{X}_{1}\right\rangle & =-\omega_{1}\left\langle Y_{1}\right\rangle \\
\left\langle\dot{Y}_{1}\right\rangle & =\omega_{1}\left\langle X_{1}\right\rangle-2 g\left\langle Z_{1} X_{2}\right\rangle \\
\left\langle\dot{Z}_{1}\right\rangle & =2 g\left\langle Y_{1} X_{2}\right\rangle \\
\left\langle Z_{1} X_{2}\right\rangle & =-2 \gamma\left\langle Z_{1} X_{2}\right\rangle-\omega_{2}\left\langle Z_{2} Y_{2}\right\rangle+2 g\left\langle Y_{1}\right\rangle \\
\left\langle Z_{1} Y_{2}\right\rangle & =\omega_{2}\left\langle Z_{2} X_{2}\right\rangle-2 \gamma\left\langle Z_{1} Y_{2}\right\rangle \\
\left\langle X_{1} X_{2}\right\rangle & =-2 \gamma\left\langle X_{1} X_{2}\right\rangle-\omega_{1}\left\langle Y_{1} X_{2}\right\rangle-\omega_{2}\left\langle X_{1} Y_{2}\right\rangle \\
\left\langle Y_{1} X_{2}\right\rangle & =-2 g\left\langle Z_{1}\right\rangle+\omega_{1}\left\langle X_{1} X_{2}\right\rangle-2 \gamma\left\langle Y_{1} X_{2}\right\rangle \\
\left\langle X_{1} Y_{2}\right\rangle & =-\omega_{2}\left\langle Y_{1} Y_{2}\right\rangle \\
& \omega_{2}\left\langle X_{1} X_{2}\right\rangle-2 \gamma\left\langle X_{1} Y_{2}\right\rangle-\omega_{1}\left\langle Y_{1} Y_{2}\right\rangle \\
\left\langle Y_{1} Y_{2}\right\rangle & =\omega_{2}\left\langle Y_{1} X_{2}\right\rangle+\omega_{1}\left\langle X_{1} Y_{2}\right\rangle-2 \gamma\left\langle Y_{1} Y_{2}\right\rangle \\
\left\langle\dot{Z}_{2}\right\rangle & =2 g\left\langle X_{1} Y_{2}\right\rangle .
\end{aligned}
$$

Having formed a useful description of the dynamics of the state in terms of a set of real parameters, $\mathbf{x}$, and identified the parameters of interest, $\mathbf{x}_{1}$, and those which are not, $\mathbf{x}_{2}$, it is straightforward to recast our problem in the input-output formalism of control theory. The system of equations above is of the form

$$
\dot{\mathbf{x}}=A \mathbf{x},
$$

but may always be written in the form

$$
\dot{\mathbf{x}}_{1}=A_{1} \mathbf{x}_{1}+B_{1} \mathbf{y}_{2},
$$




$$
\begin{aligned}
& \dot{\mathbf{x}}_{2}=A_{2} \mathbf{x}_{2}+B_{2} \mathbf{y}_{1} \\
& \mathbf{y}_{1}=C_{1} \mathbf{x}_{1}, \quad \mathbf{y}_{2}=C_{2} \mathbf{x}_{2}
\end{aligned}
$$

This explicitly formulates the full dynamics of the quantum system in terms of inputs and outputs of two systems, one of which of is of physical interest, and another for which we want to find a low-order approximation. These two systems are connected in such a way that the outputs of one system are the inputs of the other. The terms arising from the unitary interaction of the two subsystems guarantee that any quantum system will have this kind of structure where the system of interest both drives and is driven by the environment. Only in certain extreme limits where the 'environment' (the set of degrees of freedom other than the subsystem of interest) is essentially infinite-dimensional is it possible to formulate an approximate treatment for which the transfer function from inputs to the environment to outputs from the environment is essentially zero (for any finite time-horizon) and thus the outputs from the system of interest do not affect its subsequent evolution. In physics this is known as the Markov limit, and is commonly invoked in the derivation of quantum master equations [4].

Returning to our example, the system $\left(A_{2}, B_{2}, C_{2}\right)$ is both observable and controllable so it is immediately possible to find a similarity transformation for the state space that results in a balanced realization of this state space model. That is, there exists some matrix transformation $T$ that results in a transformed state space realization $\tilde{\mathbf{x}}_{2}=T \mathbf{x}_{2}, \tilde{A}_{2}=T A_{2} T^{-1}, \tilde{B}_{2}=T B_{2}, \tilde{C}_{2}=$ $C_{2} T^{-1}$ such that the associated controllability and observability gramians are equivalent and diagonal. Their diagonal elements are the Hankel singular values. This allows a balanced truncation of the model in which dimensions of the transformed state space that correspond to small Hankel singular values are simply disregarded. The resulting state space realization is also balanced and stable so long as the truncation is performed such that the smallest Hankel singular value included in the reduced state space model $\left(\bar{A}_{2}, \bar{B}_{2}, \bar{C}_{2}\right)$ is greater than the largest one that is excluded.

The error in this approximation may be tightly estimated since the transfer function corresponding to the new reduced state space model is close to the original transfer function, in the sense that the $H_{\infty}$ norm of their difference is bounded from above by twice the sum of the disregarded Hankel singular values and from below by the largest disregarded singular value. Given that our example seems to have the general flavor of a controller reduction problem, we suspect that more sophisticated techniques based on gap metric or structured singular value may be applicable [1]. However, we find that the simple balanced truncation used here is more than sufficient to find accurate approximations to the overall dynamics of our example model.
To take a concrete example, imagine that in our example system we are only concerned with the inversion of the first spin $\left\langle Z_{1}\right\rangle$. This means that we are interested in the dynamics of one of the 15 parameters defining the quantum state. The system of equations (3) shows that $\left\langle Z_{1}\right\rangle$ is coupled to only 5 of these parameters. The others may be ignored, as a simple consequence of the specific coupling we have chosen. Moreover, numerical computation of balanced realizations shows that in the broad parameter regime $g>\gamma>\omega_{1}, \omega_{2}$ only one of these parameters has a significant effect on the dynamics and so it is possible to find an approximate description of the dynamics of the environment with a state space that is only one dimensional by truncating this balanced realization. This parameter regime corresponds to the situation in which although the first spin is strongly damped the decay is very non-Markovian and so a naive adiabatic elimination is of no use (see Figure).

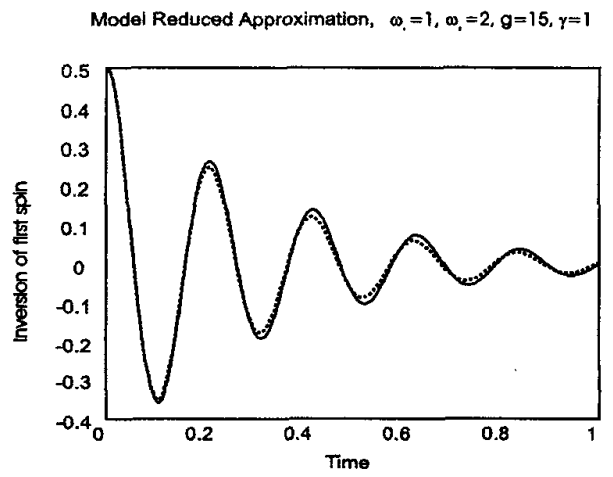

Figure 1: Numerical test of model reduction via balanced truncation for a simple quantum system: solid curve is derived from a numerical integration of the dynamical equations without approximation, dotted curve is derived from numerical integration of the reduced model.

Two advantages of this technique bear emphasizing. Firstly, the approximation is controlled in the sense that it is possible to obtain a rigorous estimate of the error resulting from truncating the balanced realization. Thus it is possible to see in advance whether a particular approximation is well justified. In other areas of the parameter space of this model the dynamics are more complicated and the radical approximation given above is not at all accurate. However, the boundaries of the simple regime are indicated by Hankel singular values which are larger and more nearly equal.

A second advantage of this method is that unlike more common (physical) approaches to modeling quantum subdynamics, there is no difficulty in dealing with entangled initial states of the system and environment. Corresponding to any initial state of the system, including all entangled states, there is some initial value of $\mathbf{x}_{1}$ 
and $\mathbf{x}_{2}$. The transformation to the balanced realization determines the appropriate initial state $\tilde{\mathbf{x}}_{2}=T \mathbf{x}_{2}$ in the state space of the balanced truncation. It makes no difference whether these vectors correspond to an entangled state or a product state of the two spins. This flexibility is in marked contrast to the situation for adiabatic elimination where it is usually necessary to assume that the system and the environment are in a product state.

\section{Applications to Quantum Error Correction}

A significant potential application of this approach to the approximate simulation of quantum systems is in the area of quantum error correction for a quantum computer. In a quantum computation quantum mechanical two-state systems or qubits replace bits as the fundamental means of storing and manipulating information. The main challenge in building a quantum computer, or simply an accurate quantum memory, is in achieving sufficient control over the state of a quantum system that it is possible to accurately perform the computation and avoid error processes. Quantum error correction provides a possible solution to the problem of random errors caused by uncontrolled couplings of the computer to its surrounding enviroment. In quantum error correction (see [5] for a technical introduction), as in its classical counterpart, the information stored in memory is protected from errors by encoding logical qubits in redundant physical degrees of freedom - these will typically be two-state systems such as those discussed above and we sometimes refer to them as physical qubits in the following.

The theory of fault-tolerant quantum computation has shown that by using encoded operations on the logical qubits and by encoding the logical qubits in many layers of coded states (concatenating codes) it is possible to perform an arbitrary computation with any desired level of accuracy given that the fundamental error rate is below some bound. Estimates exist for the value of the bound for at least some choices of code, faulttolerant gate set and error process. It is still of interest to be able to simulate the performance of errorcorrection and fault-tolerant computation in the presence of errors which are not accounted for by the code and also to find tighter bounds on the fundamental error rates necessary to achieve fault-tolerance. However, while it has been possible to simulate the evolution of simple three and five qubit codes, the full simulation of concatenated codes or fault tolerant computing schemes is not currently possible and would appear to be very challenging as a result of the exponential growth of the state space as the number of physical qubits increases. In a simple quantum code a qubit is encoded in five physical qubits, each level of concatenation will then use five qubits from the previous level in order to encode the qubit at the next highest level leading to a state space of $2^{5^{N}}$ dimensions to encode a single logical qubit, although the reduced state of the logical qubit is described by just three parameters. This is an example of a quantum mechanical system where only a relatively small amount of information about the state is of interest (the reduced state of the logical qubit) but this subsystem has non-trivial interaction with the very large state space. The application of our state-space based approach to helps firstly to identify the variables in the state space that affect the state of the logical qubit leading to a very great simplification of the model and secondly provides an approach to determining the approximate evolution of a concatenated code under various physically reasonably error processes using the kind of model reduction described above. Here we confine ourselves to describing the fundamental building blocks of the problem leaving a full treatment to further work.

In any eventual quantum computer it will be necessary to minimize couplings to the environment. These will lead to errors of the general form

$$
\rho \rightarrow \sum_{i} E_{i} \rho E_{i}^{\dagger}
$$

where, for a quantum memory, error processes correspond to $E_{i}$ different from the identity. For example, $E_{i}=X_{1}$ would flip the sign of the first qubit in the quantum memory by swapping the probability amplitudes in each of its two levels - we refer to this as a bit flip error. In a quantum error correcting scheme a logical qubit is encoded in the state of several physical qubits, the space of states of the logical qubit is then a subspace of the complete Hilbert space. The code is arranged such that the dominant error processes (for example bit flips) take the physical state into orthogonal subspaces of the total Hilbert space. Recovering from the errors then requires making a projective measurement onto these orthogonal subspaces to determine which error occured (syndrome identification) and then an appropriate recovery operation to rotate the state back into the computational subspace. The possible outcomes of this measurement correspond to projection operators $P_{i}$ onto the code and error subspaces and we will label the corresponding recovery operators $R_{i}$. The expected state of the system after the recovery operation is then

$$
\bar{\rho}=\sum_{i} R_{i} P_{i} \rho(t) P_{i} R_{i}
$$

Presuming that these operations are perfect this state is now on the code subspace and so it may be characterized by the expectation values of the corresponding logical qubit $\langle\bar{X}\rangle,\langle\bar{Y}\rangle,\langle\bar{Z}\rangle$. These correspond to particular linear combinations of the expectation values characterizing the state of the physical qubits prior to the recovery operations. 
We will consider a simple three qubit code which corrects bit-flip errors. The simplest code which corrects for all independent errors on the individual physical systems requires five physical qubits. For the bit flip code projective measurements are made of the observables $Z_{1} Z_{2}$ and $Z_{1} Z_{3}$ and the four outcomes indicate either that no error has occured or that the state of one of the physical qubits has been flipped and if so which qubit has changed. As a result the recovery operators are $X_{1}, X_{2}, X_{3}$. It is then possible to write the expectation values of the logical qubit after recovery in terms of the physical expectation values prior to recovery

$$
\begin{aligned}
\langle\bar{X}\rangle= & \left\langle X_{1} X_{2} X_{3}\right\rangle \\
\langle\bar{Y}\rangle= & \frac{1}{2}\left(\left\langle X_{1} X_{2} Y_{3}\right\rangle+\left\langle Y_{1} Y_{2} Y_{3}\right\rangle+\left\langle X_{1} Y_{2} X_{3}\right\rangle\right. \\
& \left.+\left\langle Y_{1} X_{2} X_{3}\right\rangle\right) \\
\langle\bar{Z}\rangle= & \frac{1}{2}\left(\left\langle Z_{1}\right\rangle+\left\langle Z_{2}\right\rangle+\left\langle Z_{3}\right\rangle-\left\langle Z_{1} Z_{2} Z_{3}\right\rangle\right) .
\end{aligned}
$$

This particular transformation assumes that the measurement and recovery steps are perfectly realized. This is by no means necessary, errors in either step would simply lead to a different dependence of the logical qubit expectation values on the pre-recovery expectation values. Particularly easy to take account of would be a noisy projection which would replace the projectors $\left(I \pm Z_{1} Z_{2}\right) / 2,\left(I \pm Z_{1} Z_{3}\right) / 2$ with $\left(I \pm \eta Z_{1} Z_{2}\right) / 2,(I \pm$ $\left.\eta Z_{1} Z_{3}\right) / 2$ where $\eta$ indicates the efficiency of the measurement and with $\eta=0$ the measurement results would be completely random. Similarly a noisy recovery could be simulated by replacing $\rho \rightarrow X_{i} \rho X_{i}$ by $\rho \rightarrow \eta X_{i} \rho X_{i}+(1-\eta) \rho$ as the recovery operation. These simple choices do not change the specific physical expectation values involved in the above equation just their coefficients, however, more general noise models introduce a dependence on more expectation values. In this way it is possible to identify the expectation values on the total Hilbert space which are relevant to evolution of the logical qubit given a specific measurement and recovery process.

The particular error model for the time between recovery steps determines the dynamics of the physical expectation values such as $\left\langle X_{1} X_{2} X_{3}\right\rangle$. An example of such an error model is the Lindblad master equation describing the possibility of random bit flips

$$
\begin{aligned}
\dot{\rho} & =\Gamma \mathcal{D}\left[X_{1}\right] \rho+\Gamma \mathcal{D}\left[X_{2}\right] \rho+\Gamma \mathcal{D}\left[X_{3}\right] \rho \\
& =\Gamma\left(X_{1} \rho X_{1}+X_{2} \rho X_{2}+X_{3} \rho X_{3}-3 \rho\right)
\end{aligned}
$$

This describes a situation where the physical qubits undergo independent bit flips at a constant rate. Since the code corrects for a single bit-flip, the state of the logical qubit after the correction should be unaffected by the noise to first order in time. Such a master equation could arise in the physical description of qubits subject to noisy magnetic fields affecting each of the qubits independently. In order to see how the code functions under this noise model, lets consider then the the evolution of $\langle\bar{Z}(t)\rangle$ (this is an expectation value of the logical qubit given that a recovery operation is performed at time $t$ ). The logical qubit expectation value after recovery may be related to the physical expectation values before recovery by

$$
\left(\begin{array}{c}
\langle\bar{Z}\rangle \\
\alpha
\end{array}\right)=\left(\begin{array}{cc}
\frac{1}{2} & -\frac{1}{2} \\
\frac{1}{2} & \frac{1}{2}
\end{array}\right)\left(\begin{array}{c}
Z_{T} \\
\left\langle Z_{1} Z_{2} Z_{3}\right\rangle
\end{array}\right)
$$

where $\left\langle Z_{T}\right\rangle=\left\langle Z_{1}\right\rangle+\left\langle Z_{2}\right\rangle+\left\langle Z_{3}\right\rangle$. The auxiliary degree of freedom $\alpha$ is a linear combination of the physical qubit expectation values that interacts with $\langle\bar{Z}\rangle$. None of the other degrees of freedom in the problem have an effect. The chosen error model determines the evolution of the physical qubits

$$
\frac{d}{d t}\left(\begin{array}{c}
Z_{T} \\
\left\langle Z_{1} Z_{2} Z_{3}\right\rangle .
\end{array}\right)=\left(\begin{array}{cc}
-2 \Gamma & 0 \\
0 & -6 \Gamma
\end{array}\right)\left(\begin{array}{c}
Z_{T} \\
\left\langle Z_{1} Z_{2} Z_{3}\right\rangle
\end{array}\right) .
$$

As a result it is straightforward to determine the time evolution of $\langle\bar{Z}(t)\rangle$ and $\alpha$

$$
\frac{d}{d t}\left(\begin{array}{c}
\langle\bar{Z}\rangle \\
\alpha
\end{array}\right)=\left(\begin{array}{cc}
-2 \Gamma & \Gamma \\
\Gamma & -2 \Gamma
\end{array}\right)\left(\begin{array}{c}
\langle\bar{Z}\rangle \\
\alpha
\end{array}\right)
$$

If at $t=0$ the state of the system is in the code subspace with $\langle\bar{Z}\rangle=Z_{0}$ then

$$
\langle\bar{Z}\rangle(t)=\frac{1}{2} Z_{0}\left(3 e^{-2 \Gamma t}-e^{-6 \Gamma t}\right) .
$$

As claimed, the logical qubit expectation value changes only at second order in $t$. This is true of the other expectation values also since the error model chosen here does not lead to time dependence of $\left\langle X_{1} X_{2} X_{3}\right\rangle$ and the equations for $\langle\bar{Y}\rangle$ result in the same expressions as for $\langle\bar{Z}\rangle=Z_{0}$. So we have developed here an input-output picture exactly similar to our previous discussion. Degrees of freedom describing the state of the qubit after an error correction cycle interact with a relatively small number of degrees of freedom that describe the effects of noise. Here there is only one such 'environment' variable mediating the effects of this simple noise process on the logical qubit of our particular code, but more sophisticated codes and error models will certainly lead to more complicated systems. Such a formulation of error correction is a necessary precursor the development of simple descriptions of the evolution amenable to model reductions of the kind discussed above. In particular we are interested in applying these techniques to concatenated codes and eventually fault tolerant computation.

One of the main interests of this approach is to consider the effect of errors other than those for which the code is most effective. Having determined the transformations describing syndrome detection and error recovery it is straightforward to implement the time dependence of the physical expectation values appropriate for any given error model and thereby derive the appropriate input-output model for the evolution of the logical 
qubit. One example which would be likely to be of interest in any physical implementation of the bit flip code is the situation where the errors on each physical qubit are correlated. The Lindblad master equation

$$
\dot{\rho}=\Gamma_{c} \mathcal{D}\left[X_{1}+X_{2}+X_{3}\right] \rho
$$

describes the effect of a noisy 'stray' magnetic field which affects all three of the qubits. In this case the equations for the time evolution of $\left\langle Z_{1} Z_{2} Z_{3}\right\rangle$ and $\left\langle Y_{1} Y_{2} Y_{3}\right\rangle$ couple to other expectation values rather than simply damping as they do in the original model. As in the first error model the differential equations for the logical qubit expectation values couple to variables describing the state of the environment - in this case it turns out that there are two such degrees of freedom and the resulting evolution of the logical qubit has a first order time dependence proportional to $\Gamma_{c}$.

In concatenated coding there are several levels of error correction. The logical qubits described above are in turn combined into triples which code for a logical qubit at a higher level. The measurement and correction transformations are applied firstly to the triples of physical qubits at the lowest level exactly as described above and then at each higher level of the code. Thus for a single concatenation of the above code there are nine physical qubits and the overall tranformation between the top level logical qubit and the physical qubits may be derived by applying the previous formula to each level of the code and is of the form

$$
\begin{aligned}
\langle\bar{Z}\rangle= & \frac{1}{4}\left(\sum_{i=1}^{9}\left\langle Z_{i}\right\rangle-\sum_{i, j, k} c_{i j k}^{(3)}\left\langle Z_{i} Z_{j} Z_{k}\right\rangle\right. \\
& +\sum_{i, j, k, l, m} c_{i j k l m}^{(5)}\left\langle Z_{i} Z_{j} Z_{k} Z_{l} Z_{m}\right\rangle \\
& -\sum_{i, j, k, l, m, n, o} c_{i j k l m n o}^{(7)}\left\langle Z_{i} Z_{j} Z_{k} Z_{l} Z_{m} Z_{n} Z_{o}\right\rangle \\
& \left.+\left\langle Z_{1} Z_{2} Z_{3} Z_{4} Z_{5} Z_{6} Z_{7} Z_{8} Z_{9}\right\rangle\right)
\end{aligned}
$$

for a set of coefficients $c^{(i)}$. Each of the five terms in the above sum decays at a given rate under the first error model discussed above and this means that it is necessary to keep track of only five degrees of freedom in this concatenated code in order to determine the evolution of $\langle\bar{Z}\rangle$ - the value of $\langle\bar{Z}\rangle$ itself and four degrees of freedom that mediate the effects of noise on the logical qubit. So there are only eight degrees of freedom (including those necessary to solve for $\langle\bar{Y}\rangle$ ) that are in fact coupled to the state of the logical qubit even though there are 511 degrees of freedom in the full quantum state. The scaling in the size of the problem for this particular error model is $3^{n}$ where $n$ is the number of levels of concatenation which is more favourable than the scaling of the entire Hilbert space $2^{3^{n}}$.
In general the protection of a quantum memory will consist of periods where the quantum state is left to decohere followed by the application of a possibly noisy error detection and correction. As in the previous section we have formulated the description of this evolution in terms of a logical qubit with an input-output coupling to a certain state-space model describing the effects of noise on the logical qubit. The resulting systems are analytically simple for individual codes and simple error models, however the dimensionality of the problem grows with levels of concatenation of the code and we anticipate that model reduction techniques will be useful in obtaining tractable models for the overall evolution. So far we have discussed the development of description of a single decoherence-recovery iteration. This overall transformation, once determined, is applied repeatedly to describe the build-up of errors in the quantum memory. This is a discrete time model which may itself be amenable to some form of model reduction. It remains for further work however to develop parallel techniques for describing the interaction of logical qubits during a computation and thus address the question of fault tolerant computation itself.

\section{Conclusions}

In this paper we have briefly outlined the initial stages in a program of applying the analysis techniques of robust control theory to quantum systems. We believe that these techniques will have broad application and in particular will enable the simulation of the dynamics of a quantum mechanical memory that employs a highly concatenated quantum error correcting code and perhaps also of a fault-tolerant quantum computation. The application of both analysis and synthesis techniques taken from robust control theory will play an important role in the development of emerging quantum technologies, such as quantum computation.

\section{References}

[1] K. Zhou and J. C. Doyle, Essentials of Robust Control (Prentice Hall, New Jersey, 1998).

[2] J. Preskill, Phys. Today 52, 24 (1999).

[3] H. Mabuchi, J. Ye and H. J. Kimble, Appl. Phys. B 65, 1095 (1999); C. J. Hood, T. W. Lynn, A. C. Doherty, A. S. Parkins, H. J. Kimble, Science 287, 1447 (2000).

[4] D. F. Walls, G. J. Milburn, Quantum Optics (Springer-Verlag, Berlin, 1994).

[5] Daniel Gottesman, An Introduction to Quantum Error Correction quant-ph/0004072 (2000); Daniel Gottesman, The Heisenberg Representation of Quantum Computers quant-ph/9807006 (1998) and references therein. 